\title{
Efficacy and safety of enasidenib and azacitidine combination in patients with IDH2 mutated acute myeloid leukemia and not eligible for intensive chemotherapy
}

\author{
Sangeetha Venugopal (iD ${ }^{1}$, Koichi Takahashi (D) ${ }^{1}$, Naval Daver (D) ${ }^{1}$, Abhishek Maiti ${ }^{1}$, Gautam Borthakur (iD ${ }^{1}$, Sanam Loghavi (iD) ${ }^{2}$, \\ Nicholas. J. Short (iD ${ }^{1}$, Maro Ohanian ${ }^{1}$, Lucia Masarova ${ }^{1}$, Ghayas Issa (D) ${ }^{1}$, Xuemei Wang ${ }^{3}$, Bueso-Ramos Carlos ${ }^{2}$, Musa Yilmaz iD ${ }^{1}$, \\ Tapan Kadia (D) ${ }^{1}$, Michael Andreeff (iD) ${ }^{1}$, Farhad Ravandi ${ }^{1}{ }^{1}$, Marina Konopleva (D) ${ }^{1}$, Hagop M. Kantarjian (D) ${ }^{1}$ and \\ Courtney D. DiNardo (iD ${ }^{1 凶}$
}

(c) The Author(s) 2022

Preclinically, enasidenib and azacitidine (ENA + AZA) synergistically enhance cell differentiation, and venetoclax (VEN), a small molecule $\mathrm{BCl} 2$ inhibitor (i) is particularly effective in IDH2 mutated acute myeloid leukemia (IDH2 ${ }^{\text {mut }} \mathrm{AML}$ ). This open label phase II trial enrolled patients (pts) with documented IDH2 ${ }^{\text {mut }} \mathrm{AML}$. All patients received AZA $75 \mathrm{mg} / \mathrm{m}^{2} / \mathrm{d} \times 7 \mathrm{~d} / \mathrm{cycle}$ and ENA $100 \mathrm{mg}$ QD continuously. Concomitant Bcl2i and FLT3i were allowed (NCT03683433).Twenty-six pts received ENA + AZA (median 68 years, range, 24-88); 7 newly diagnosed (ND) and 19 relapsed/refractory (R/R). In R/R AML patients, three had received prior ENA and none had received prior VEN. The composite complete remission rate (CRC) [complete remission (CR) or complete remission with incomplete hematologic recovery (CRi)] was $100 \%$ in ND AML, and $58 \%$ in R/R AML. Median OS was not reached in ND AML with median follow-up of 13.1 months (mo); Pts treated in first relapse had improved OS than those with $\geq 2$ relapse (median OS not reached vs 5.2 mo; HR $0.24,95 \% \mathrm{Cl} 0.07-0.79, p=0.04$ ). Two patients received ENA + AZA with a concomitant FLT3i, one responding ND AML patient and one nonresponding R/R AML patient. Seven R/R AML pts received ENA + AZA + VEN triplet, and with median follow up of 11.2 mo, median OS was not reached and 6-mo OS was $70 \%$. The most frequent treatment-emergent adverse events include febrile neutropenia (23\%). Adverse events of special interest included all-grade IDH differentiation syndrome (8\%) and indirect hyperbilirubinemia (35\%). ENA + AZA was a well-tolerated, and effective therapy for elderly pts with $I D H 2^{\text {mut }} \mathrm{ND} A M L$ as well as pts with R/R AML. The addition of VEN to ENA + AZA appears to improve outcomes in R/R IDH2 ${ }^{\text {mut }} \mathrm{AML}$.

Clinical trial registration information: https://clinicaltrials.gov/.NCT03683433

Blood Cancer Journal (2022)12:10; https://doi.org/10.1038/s41408-021-00604-2

\section{INTRODUCTION}

Until recently, older patients with acute myeloid leukemia (AML) not eligible for intensive chemotherapy were treated with azacitidine (AZA), demonstrating response rates of $25-31 \%$ and a median overall survival (OS) under 12 months in newly diagnosed (ND) patients [1-4]. In November 2018, venetoclax (VEN), a small molecule $\mathrm{BCl} 2$ inhibitor (i) in combination with hypomethylating agent (HMA: AZA or decitabine) was approved for the treatment of ND AML in patients aged $\geq 75$ years and/or not eligible for intensive chemotherapy. ND AML patients treated with VEN + AZA demonstrated $66.4 \%$ composite complete remission rate $(\mathrm{CRC})$ [complete remission (CR) or complete remission with incomplete hematologic recovery (CRi)] and a median OS of 14.7 months, with particularly favorable outcomes in those harboring isocitrate dehydrogenase 2 (IDH2) gene mutations [5]. Despite these marked strides in the treatment of $A M L$, the majority of patients with AML still experience relapse, emphasizing the unmet need for effective therapies adept at inducing durable remission [6].

IDH2 mutations (IDH2 ${ }^{\text {mut }}$ )occur in approximately $12-15 \%$ of patients with $A M L$, and occur with increasing frequency in older patients [7]. Wild-type IDH2 catalyzes the conversion of isocitrate to a-ketoglutarate in the Krebs cycle; whereas, mutant IDH2 neomorphically generates the oncometabolite 2-hydroxyglutarate $(2-\mathrm{HG})$, leading to DNA hypermethylation and myeloid cell differentiation arrest, promoting leukemogenesis [8]. Enasidenib (ENA), a first-in-class small molecule mutant IDH2i is approved as monotherapy in R/R IDH2 ${ }^{\text {mut }} \mathrm{AML}$ [9]. In the R/R setting, ENA ( $n=$ 171) demonstrated an overall response rate [ORR, including $C R$, $\mathrm{CRi}$, partial remission (PR), and morphologic leukemia-free state (MLFS)] of $40.3 \%$, with $19.3 \%$ CR, and a median OS of 8.8 months [9]. In a smaller cohort of ND patients with IDH2 ${ }^{\text {mut }}$ AML $(n=39)$ treated within the same study, ENA showed an ORR of $30.8 \%$, including $18 \% \mathrm{CR}$, and a median OS of 11.3 months [10].

\footnotetext{
${ }^{1}$ Department of Leukemia, The University of Texas MD Anderson Cancer Center, Houston, TX, USA. ${ }^{2}$ Department of Hematopathology, The University of Texas MD Anderson
} Cancer Center, Houston, TX, USA. ${ }^{3}$ Department of Biostatistics, The University of Texas MD Anderson Cancer Center, Houston, TX, USA. ${ }^{\circledR}$ email: cdinardo@mdanderson.org

Received: 27 September 2021 Revised: 10 December 2021 Accepted: 20 December 2021

Published online: 25 January 2022 
Preclinically, ENA + AZA combination synergistically promoted differentiation, and potentiated leukemic cell death [11]. Interim results of the ongoing randomized study evaluating ENA + AZA in patients with ND IDH2 ${ }^{\text {mut }} \mathrm{AML}$ reported a favorable ORR of $71 \%$, with $53 \% \mathrm{CR}$ rates and a median OS of 22 months [12]. The effectiveness of this combination in the $\mathrm{R} / \mathrm{R} I D H 2^{\text {mut }} \mathrm{AML}$ is unknown. Additionally, the $\mathrm{BCl}$ 2-inhibitor venetoclax (VEN) is particularly effective in $I D H 2^{\text {mut }} \mathrm{AML}$ as the accumulation of the oncometabolite 2-HG inhibits cytochrome $\mathrm{C}$ oxidase and effectively primes $\mathrm{AML}$ blasts to $\mathrm{Bcl} 2$ inhibition. Preclinically, ENA + VEN combination demonstrated improved survival in murine models, as compared to either agent alone or with sequential use of the two agents $[13,14]$. Herein, we report our experience with the combination of ENA + AZA as frontline or salvage AML therapy (NCT03683433) for IDH2 ${ }^{\text {mut }} \mathrm{AML}$, with or without the addition of VEN.

\section{Study design and participants}

This single-center, phase 2 trial enrolled patients with $I D H 2^{\text {mut }} \mathrm{AML}$ and [1] age $\geq 60$ years with ND AML and ineligible for intensive chemotherapy [2], age $>18$ years with treated or untreated secondary AML arising from an antecedent hematologic neoplasm [15] and/or [3] R/R AML. Eligibility required an Eastern Cooperative Oncology Group (ECOG) Performance Status $\leq 3$ and adequate endorgan reserve. Prior treatment with ENA, AZA, and/or VEN was allowed. The study was approved by the Institutional Review Board at the University of Texas, MD Anderson Cancer Center and conducted in accordance with the Declaration of Helsinki.

\section{Procedures}

Patients received daily AZA (IV or SQ $75 \mathrm{mg} / \mathrm{m}^{2}$ x 7 days) and ENA $100 \mathrm{mg}$ orally continuously per 28-day cycle. Cytoreduction with hydroxyurea and/or 1 dose of cytarabine (up to $2 \mathrm{~g} / \mathrm{m}^{2}$ ) prior to treatment initiation was allowed. As $I D H 2^{\text {mut }}$ are known to frequently co-occur with activating $F L T 3^{\text {mut }}$ [7], approved FLT3i such as sorafenib, gilteritinib or midostaurin were allowed, as clinically appropriate [16]. Similarly, the addition of VEN in combination with AZA has been shown to be particularly effective in patients with $I D H 2^{\text {mut }} \mathrm{AML}$; therefore, the addition of VEN was allowed in combination with ENA and AZA, as clinically indicated $[5,17]$. Patients remained on study until disease progression, unacceptable toxicity, or transition to allogeneic hematopoietic stem cell transplant (HSCT). All adverse events (AE) were monitored during the study protocol and categorized as per the Common Terminology Criteria for Adverse Events (CTCAE version 4.03)

\section{Outcomes}

The primary outcome of this phase II trial was to assess the efficacy as measured by the composite $C R$ rate $(C R c=C R+C R i)$ within the first four cycles of therapy. Secondary outcomes included OS, Event-free survival (EFS), duration of response (DoR), and toxicity. OS was measured from the start of treatment until the date of death due to any cause or censored at the date of the last follow-up. EFS was measured from the date of treatment start until treatment failure, relapse, or death from any cause, whichever occurred first. Patients who were alive and without disease relapse at the time of the last follow-up were censored. Duration of response is defined, among responders, as the time duration between the date of response and the date of disease relapse or death from any cause, whichever occurred first. Patients who were alive and without disease relapse at the time of the last follow-up were censored. Survival endpoints were not censored at HSCT.

\section{Exploratory outcomes}

Immunophenotypic evidence of measurable residual disease (MRD) was performed using eight-color flow cytometry. MRD was quantified as percentage of total leukocytes after the exclusion of most red blood cell precursors. At least 200,000 events were acquired to achieve a minimum sensitivity of $10^{-3}-10^{-4}(0.1-0.01 \%)$, as described previously [18]. Nextgeneration sequencing (NGS) was employed for the assessment of mutations. The assay interrogates the entire exonic or hotspot regions of 81 genes that are frequently mutated in myeloid malignancies, as described previously [19]. A minimum sequencing coverage of $\times 250$ was used to reach an analytical sensitivity of $1 \%$ mutant reads in a background of wild-type. FLT3 mutation analysis was performed using a multiplex fluorescent-based polymerase chain reaction (PCR) analysis followed by capillary electrophoresis.

Pre-specified exploratory objectives included an analysis of the relationship between co-occurring mutations, rate of $I D H 2$ mutation clearance by NGS, the incidence of MRD-negativity by flow cytometry, and the incidence and characteristics of IDHiassociated differentiation syndrome (IDH-DS) with combination therapy.

\section{Statistical Considerations}

This study was not designed for comparison. We focused on the estimation of activity of the combination therapy, using the method of Thall, Simon, and Estey for futility and toxicity monitoring [20]. The trial was designed with a maximum sample size of 50 . With this sample size, the half-width of the $95 \%$ confidence interval will be at most 0.14 when estimating the $\mathrm{CRc}$ rate. For the purpose of futility monitoring, our target CRc rate is $40 \%$ (desirable), while a CRc rate of $30 \%$ or lower will be considered not desirable. The Bayesian interim futility monitoring will be applied in cohort size of 10 and if at any interim look, we determine that there is less than $5 \%$ chance that the $\mathrm{CRc}$ rate improves over historical rate by more than $10 \%$, the trial will be stopped early due to futility. Based on this stopping rule, the trial will be stopped early if we observe $</=1$ out of 10,4 out of 20,7 out of 30, or 11 out of 44 patients with CRc. Similarly, we will implement the Bayesian toxicity monitoring rule in cohort size of 10 and the trial will be stopped early if, at any interim look, there is more than $90 \%$ chance that the toxicity rate is more than $30 \%$. No early futility or toxicity stopping rules were met and the study continues to enroll.

All patients who received at least one dose of the combination therapy were evaluable for safety and response assessments regardless of the duration of treatment. Patient characteristics were summarized using frequency (percentage) for categorical variables and median (range) for continuous variables. Toxicity type and severity were summarized. Time-to-event endpoints including OS, EFS, and DoR were estimated using the method of Kaplan-Meier and log-rank tests were used to compare between subgroups. All analyses were done using Prism, GraphPad (version 8.4).

\section{RESULTS}

\section{Patient characteristics}

Twenty-six patients were enrolled between September 20, 2018 and October 7, 2020, (ND = 7, R/R=19). The consort flowchart is shown in Fig. 1, and baseline characteristics in Table 1

Among patients with ND IDH2 ${ }^{\text {mut }} \mathrm{AML}(n=7)$, the median age was 77 years (range, 66-81); one patient had prior HMA exposure for an antecedent hematological neoplasm. Among those with R/R IDH2 ${ }^{\text {mut }}$ AML $(n=19)$, the median age was 64 years (range, 24-88). Eight patients were in first relapse (42\%) and eleven (58\%) in the second relapse or beyond. Patients with R/R AML had received a median of two prior therapies (range,1-4) of which nine (47\%) had prior HMA and three (16\%) had prior ENA exposure, two patients had received prior intensive chemotherapy (11\%) and five (26\%) had relapsed post-HSCT. None had prior VEN exposure. 


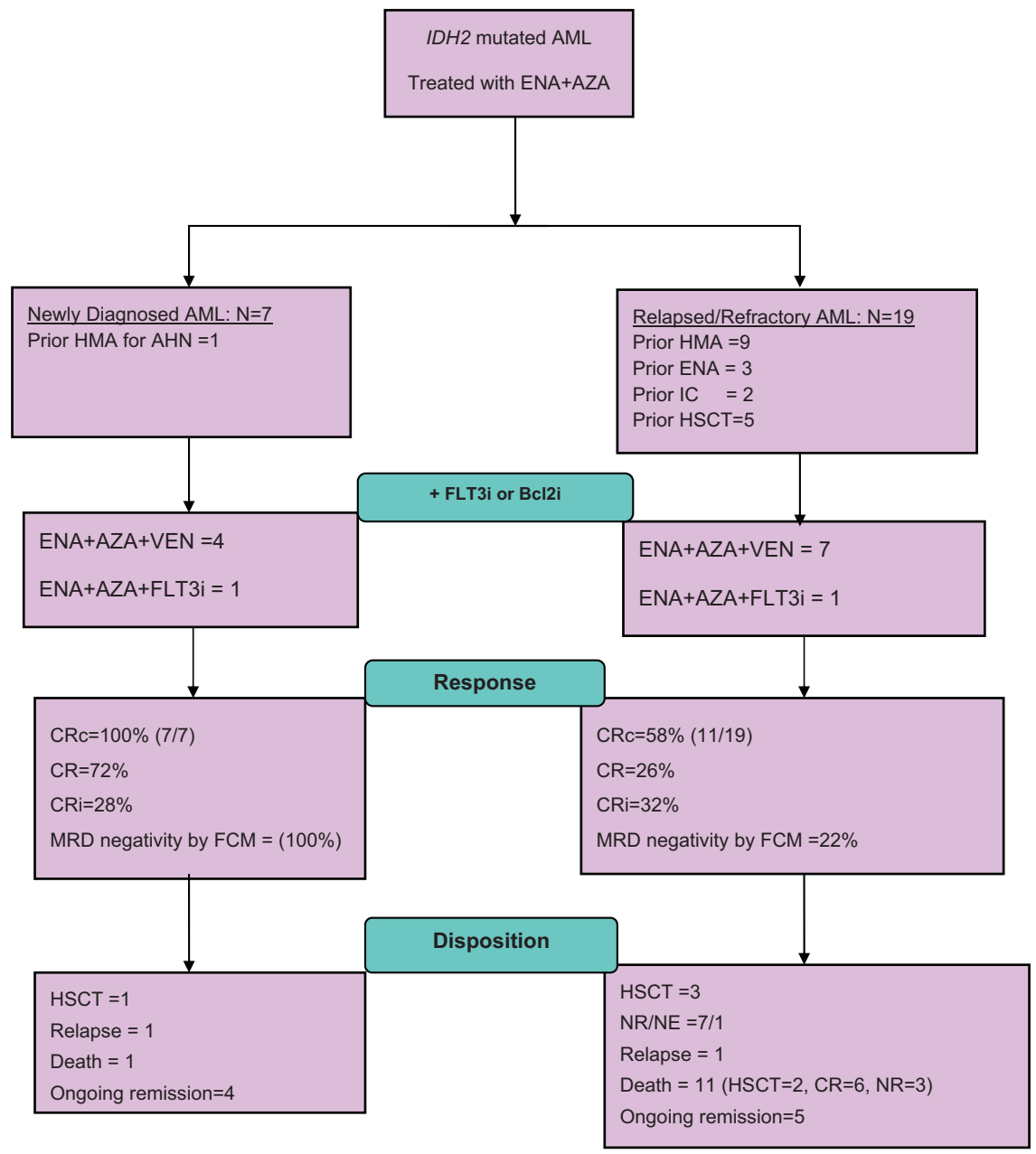

Fig. 1 Consort diagram of Phase II trial of ENA+AZA combination in IDH2 ${ }^{\text {mut }}$ AML. Consort diagram. AML- Acute myeloid leukemia, ENAEnasidenib, AZA- Azacitidine, HMA- hypomethylating agent, AHN- antecedent hematological neoplasm, IC- intensive chemotherapy, HSCTHematopoietic stem cell transplant, i- inhibitor, VEN- venetoclax, CRc- composite complete remission, CR- complete remission, CRi- complete remission with incomplete hematological recovery, MRD- measurable residual disease, FCM -Flowcytometry, NR -no response, NE- not evaluable.

Among patients with ND IDH2 ${ }^{\text {mut }} \mathrm{AML}, 5(71 \%)$ were categorized as intermediate risk and two patients (29\%) with adverse risk according to ELN 2017 [21]; all seven (100\%) patients had AML with IDH2 p.R140Q with a median variant allele frequency (VAF) of $42 \%$ (range, 26-50\%). This cohort was enriched in co-occurring mutations in SRSF2 $(71 \%, n=5)$, and FLT3 $(29 \%, n=2$ one internal tandem duplication (ITD) and one tyrosine kinase domain point mutation (TKD); no concurrent TP53 $3^{\text {mut }}$ were detected. Contrastingly in $\mathrm{R} / \mathrm{R} I D H 2^{\text {mut }} \mathrm{AML}$, ELN adverse risk was more frequent (53\%) compared with intermediate risk (47\%). Among patients with R/R AML, 14 (74\%) harbored IDH2 p.R140Q and 5 (26\%) had IDH2 p.R172K mutations with median VAFs of $23 \%$ (range, 3-50\%), and $35 \%$ (range, 18-46\%), respectively. The R/R cohort was notable for concurrent mutations involving RUNX1 (21\%), KRAS/ NRAS (16\%), TP53 (11\%), and FLT3 -ITD (5\%).

\section{Efficacy}

Twenty-five patients were evaluable for response, summarized in Table 2. Among patients with ND IDH2 ${ }^{\text {mut }} \mathrm{AML}$, the $\mathrm{CRc}(\mathrm{CR} / \mathrm{CRi})$ was $100 \%(n=7)$ with $72 \%(n=5)$ achieving a CR. Among the two patients who achieved CRi, one patient (50\%) achieved hematological improvement in both hemoglobin and platelets. All seven patients (100\%) additionally achieved MRD negativity by flow cytometry (MRD neg $F C M)$. Four patients received concurrent venetoclax (ENA + AZA + VEN), and one patient with comutated
FLT3-ITD received concurrent gilteritinib. One patient (14\%) underwent $\mathrm{HSCT}$ in $\mathrm{MRD}^{\text {neg }} \mathrm{FCM}$ remission; notably, this patient had prior HMA exposure and had received the "triplet" of ENA + $A Z A+V E N$. Among the responders, the median duration of response was not reached (range, 1.6-not reached) in the entire cohort and in the four patients receiving the ENA + AZA + VEN triplet and was 7.5 months in the one patient receiving the ENA + AZA + gilteritinib triplet. At a median follow-up of 13.1 months, median OS and EFS were not reached, and the 1-year OS and EFS rates were $83 \%$ and $56 \%$, respectively.

Among 19 patients with R/R IDH2 ${ }^{\text {mut }} \mathrm{AML}, 18$ were evaluable for response, the CRc was $61 \%(n=11)$ with $28 \%(n=5)$ achieving a CR. Hematological improvement was not noted in this heavily pretreated cohort. The MRD ${ }^{\text {neg }}$ FCM rate was $22 \%(n=2 / 9)$ among the responding patients; notably both of them received ENA + AZA + VEN triplet combination. Seven patients were refractory to treatment including a patient with FLT3-ITD co-mutation with relapsed $A M L$ post-HSCT and received ENA + AZA + midostaurin, and another pt who received ENA + AZA + VEN triplet combination. Among the eleven responders, six patients received ENA + $A Z A+V E N$, among which five had prior HMA exposure, and one had prior ENA exposure. The CRc in patients treated with ENA + $A Z A+$ VEN was $86 \%(n=6 / 7)$ with a CR rate of $71 \%(n=5 / 7)$. Among patients with ECOG $\geq 2$, the CRc rate was $80 \%(n=4 / 5)$, and among those with prior HMA, ENA and HSCT exposure, the 
Table 1. Baseline Demographics.

\begin{tabular}{|c|c|c|c|}
\hline $\begin{array}{l}\text { Baseline } \\
\text { Characteristics }\end{array}$ & $\begin{array}{l}\text { Total } \\
(n=26)\end{array}$ & $\begin{array}{l}\text { Newly } \\
\text { diagnosed } \\
n=7\end{array}$ & $\begin{array}{l}\text { Relapsed/ } \\
\text { refractory } \\
n=19\end{array}$ \\
\hline Age (years) & $68(24-88)$ & $77(66-81)$ & $64(24-88)$ \\
\hline Sex (male) & $20(84)$ & $6(86)$ & $14(82)$ \\
\hline \multicolumn{4}{|l|}{ Performance status } \\
\hline ECOG 0 & $1(4)$ & $1(14)$ & 0 \\
\hline ECOG 1 & $18(69)$ & $4(57)$ & $14(74)$ \\
\hline$E C O G \geq 2$ & $7(28)$ & $2(29)$ & $5(26)$ \\
\hline \multicolumn{4}{|c|}{ Hematological parameters } \\
\hline $\begin{array}{l}\text { Hemoglobin (mg/ } \\
\text { dL) }\end{array}$ & $\begin{array}{l}8.6 \\
(6.2-13.7)\end{array}$ & $8.4(6.2-10.6)$ & $8.8(7.1-13.7)$ \\
\hline $\begin{array}{l}\text { White Blood } \\
\text { Count, } \times 10^{9} / \mathrm{L}\end{array}$ & $\begin{array}{l}3.8 \\
(0.4-270)\end{array}$ & $6(2-270)$ & $1.5(0.4-36)$ \\
\hline Platelet, $\times 10^{9} / \mathrm{L}$ & $\begin{array}{l}52 \\
(12-1145)\end{array}$ & $74(30-1145)$ & $30(12-223)$ \\
\hline $\begin{array}{l}\text { Circulating } \\
\text { blasts (\%) }\end{array}$ & $20(0-95)$ & $34(10-95)$ & $5(0-66)$ \\
\hline $\begin{array}{l}\text { Bone marrow } \\
\text { blasts (\%), }\end{array}$ & 39 [4-87] & $57[20-86]$ & 20 [4-87] \\
\hline \multicolumn{4}{|c|}{ ELN 2017 risk group } \\
\hline Intermediate & $14(59)$ & $5(71)$ & $9(47)$ \\
\hline Adverse & $12(41)$ & $2(29)$ & $10(53)$ \\
\hline \multicolumn{4}{|l|}{ Diagnosis } \\
\hline In first relapse & & 0 & $8(42)$ \\
\hline $\begin{array}{l}\text { In second or later } \\
\text { relapse }\end{array}$ & & 0 & $11(58)$ \\
\hline \multicolumn{4}{|c|}{ Prior AML therapies } \\
\hline HMA & $9(28)$ & $1(14)$ & $9(42)$ \\
\hline ENA & $3(12)$ & & $3(16)$ \\
\hline $\mathrm{HSCT}$ & $5(19)$ & & $5(26)$ \\
\hline \multicolumn{4}{|c|}{ Co-occurring mutations } \\
\hline ASXL1 & $5(19)$ & $2(29)$ & $3(16)$ \\
\hline DNMT3A & $8(31)$ & $2(29)$ & $6(31)$ \\
\hline FLT3 & $3(12)$ & $2(29)$ & $1(5)$ \\
\hline NPM1 & $5(19)$ & $1(14)$ & $4(21)$ \\
\hline$(K / N) R A S$ & $3(12)$ & 0 & $3(16)$ \\
\hline RUNX1 & $6(23)$ & $2(29)$ & $4(21)$ \\
\hline SRSF2 & $10(38)$ & $5(71)$ & $5(26)$ \\
\hline TET2 & $5(19)$ & $1(14)$ & $4(21)$ \\
\hline TP53 & $2(8)$ & 0 & $2(11)$ \\
\hline
\end{tabular}

ECOG- Eastern Cooperative Oncology Group, ELN- European Leukemia Net, HMA- Hypomethylating agent, ENA- Enasidenib, HSCT- Hematopoietic cell transplantation.

All results expressed as No. (\%) or median [Minimum-maximum], unless specified.

CRc rates were $67 \%(n=6 / 9), 100 \%(n=3 / 3)$, and $60 \%(n=3 / 5)$ respectively. Patients in first relapse had numerically higher $\mathrm{CRc}$ $(75 \%, n=6 / 8)$ than those in second relapse or higher (CRc: $50 \%$, $n=5 / 10)$. Three patients (17\%) underwent HSCT, among which two were in second relapse or higher.

Among the responders, the median duration of response was not reached. At a median follow up of 13.1 months, median EFS was 6.9 months with a 1-year EFS rate of $25 \%$. Among the responders in the $\mathrm{R} / \mathrm{R}$ cohort, the median OS was 9.7 months with a 1-year OS rate of $32 \%$, and the median OS was not reached when censored for SCT. Two patients had early
Table 2. Outcomes in newly diagnosed and relapsed/refractory patients with $I D H 2$ mutant acute myeloid leukemia.

\begin{tabular}{|lll|}
\hline Response & $\begin{array}{l}\text { Newly } \\
\text { diagnosed } \boldsymbol{n}=\mathbf{7}\end{array}$ & $\begin{array}{l}\text { Relapsed/ } \\
\text { refractory } \boldsymbol{n}= \\
\mathbf{1 9}\end{array}$ \\
\hline $\mathrm{CRC}$ & $7(100)$ & $11(58)$ \\
\hline $\mathrm{CR}$ & $5(72)$ & $5(26)$ \\
\hline $\mathrm{CRi}$ & $2(28)$ & $6(32)$ \\
\hline MRD negativity by FCM & $7 / 7(100)$ & $2 / 9(22)$ \\
\hline Not evaluable & 0 & $1(5)$ \\
\hline $\begin{array}{l}\text { No response } \\
\text { Median number of cycles }\end{array}$ & 0 & $7(37)$ \\
given (range) & $3(1-8)$ & $4(1-17)$ \\
\hline $\begin{array}{l}\text { Median time to best } \\
\text { response, months (range) }\end{array}$ & $1.6(1.0-4.2)$ & $1.8(0.8-5.4)$ \\
\hline
\end{tabular}

$C R C$-composite complete remission rate $=C R+C R i, C R$ - complete remission, $C R i$-CR with incomplete hematologic recovery, MRD- measurable residual disease, FCM- flowcytometry

All results expressed as No. (\%) or median [Minimum-maximum], unless specified.

mortality in the post-SCT period due to infection and graft versus host disease. Patients treated in first relapse had a significantly superior OS than those treated in second or later relapse (median OS not reached vs 5.2 mo; 1 -year OS $75 \%$ vs $10 \%, \mathrm{HR} 0.24,95 \% \mathrm{Cl} 0.07-0.79, \mathrm{P}=0.04$ ) (Fig. 2). In patients treated with ENA + AZA + VEN, at a median follow up of 11.2 months, median OS and EFS were not reached and 6-mo OS and EFS were $70 \%$ and $67 \%$, respectively. Although the sample size is small, R/R AML patients treated with ENA + AZA + VEN exhibited a trend towards better OS than those treated with ENA + AZA (median OS not reached vs 6.0 months, 1-year OS $67 \%$ vs $20 \%$, HR $0.29,95 \% \mathrm{Cl} 0.09-0.97 \mathrm{P}=0.08$ ) (Fig. 3 ).

\section{Exploratory outcomes}

Co-occurring mutational patterns with response and treatment details are shown in Fig. 4. Variant allele frequency (VAF) of $\mathrm{IDH} 2^{\text {mut }}$ at the start of treatment was available in 22 patients and ranged from $3 \%$ to $50 \%$. The median VAF of $I D H 2^{\text {mut }}$ did not differ greatly between p.R140Q $(n=17)$ and p.R172K $(n=5)$ variants (36\% and 32\%, respectively). Sequential NGS was available for 16 patients $(N D=5, R / R=11)$ at the time of response evaluation. Persistent $I D H 2^{\text {mut }}$ was detected in all patients $(n=16)$. Among all responding patients with $\mathrm{ND} I D H 2^{\text {mut }} \mathrm{AML}$, the median VAF decrease was greater than $50 \%$ (median decrease of $42 \%$ to $18 \%$ ). Among the responding patients with $\mathrm{R} / \mathrm{R} I D H 2^{\text {mut }} A M L$, the median VAF decreased from $32 \%$ to $19 \%$. In R/R IDH $2^{\text {mut }} A M L$, four evaluable patients with p.R172K variant achieved CR/CRi and had numerically better survival compared to those with p.R140Q variant ( 11.4 vs 5.2 months, 6 -mo OS $75 \%$ vs $49 \%, P=0.36$ ). While flow MRD negativity was common, no responding patients attained $I D H 2$ negative status by NGS (sensitivity $1 \%$ ).

Common co-occurring mutations among the entire cohort involved SRSF2 (38\%), DNMT3A (31\%), and RUNX1 (23\%) followed by NPM1, ASXL1, TET2 (20\% each). KRAS/NRAS (24\%) and TP53 (8\%) mutations were exclusively seen in the R/R group. Patients with ND AML had fewer number of mutations (median $=3$, range $1-7$ ) than the $R / R$ group (median $=4$, range $1-7$ ) with no difference between responders and non-responders. Molecular subgroups conferring treatment resistance to ENA $[22,23]$ [KRAS/NRAS (86\%), SRSF2 (57\%), ASXL1 (57\%), DNMT3A, RUNX1, and BCOR(14\% each)] were enriched in non-responders. The CRc rate was $64 \%$ in patients without KRAS/NRAS and TP53 mutations. In contrast, the CRc rate was only $40 \%$ in patients with KRAS/NRAS and/or TP53 mutations. 


\section{Safety}

All patients were evaluable for safety. The median number of treatment cycles received was three (range, $0-18$ ) including four in responding and three in non-responding patients. ENA + AZA combination was reasonably well tolerated (Table 3 ). Thirty- and

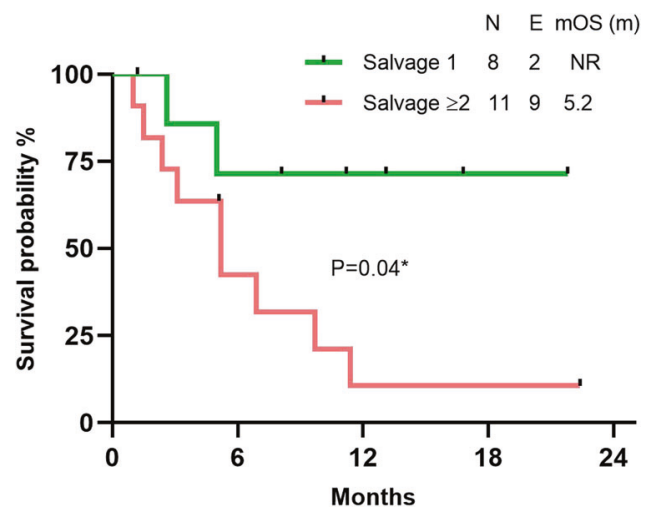

Fig. 2 Kaplan-Meier plot showing overall survival (OS) by line of therapy in R/R cohort. $\mathrm{N}$ number, $\mathrm{E}$ events, $\mathrm{mOS}(\mathrm{m})$ median overall survival in months.

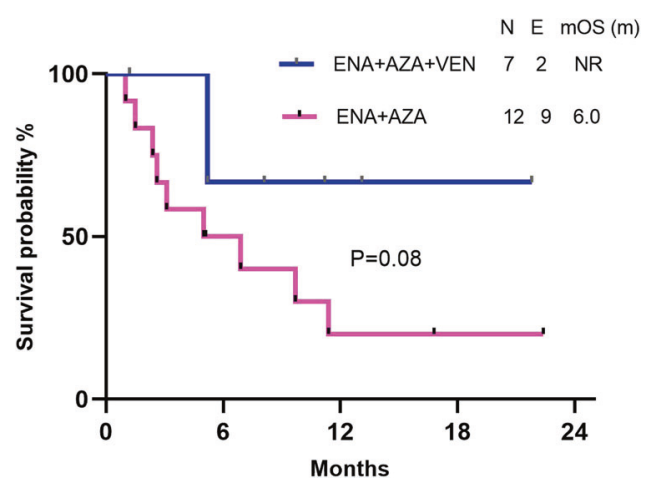

Fig. 3 Kaplan-Meier plot showing overall survival (OS) by venetoclax status in R/R cohort. $\mathrm{N}$ number, $\mathrm{E}$ events,mOS $(\mathrm{m})$ median overall survival in months.
60 - day mortality rates were $0 \%$ each in the ND group and $5 \%$ each ( $n=1$, each) in the R/R group. At the time of data cut off, eight patients $(31 \%)$ remain on treatment, and reasons for treatment discontinuation include $\mathrm{HSCT}(n=4,15 \%)$, disease progression $(n=4,15 \%)$, and death $(n=10$ : disease progression $=5$, relapse $=4, C R i=1$ ).

Treatment-emergent adverse events $(T E A E) \leq$ grade 3 were reported in $22 / 26$ pts (85\%). Most frequent grade $\leq 3$ TEAEs include febrile neutropenia (23\%), and indirect hyperbilirubinemia (35\%). Gastrointestinal AEs of any grade included vomiting (11\%) and diarrhea (21\%). Tumor lysis syndrome $(0 \%)$ was not observed in patients treated with ENA + AZA + VEN combination. Most TEAEs were manageable without dose interruption.

IDH-DS was reported in 2 pts (8\%) of which one received concurrent gilteritinib for $F L T 3 / I D H 2^{\text {comutated }}$ AML. In this patient, IDH-DS (leukocytosis, grade 1 creatinine increase) was observed on D18 of treatment and was managed with steroids with normalization of kidney function. Further treatment was continued without dose interruption or modifications. ENA was held in the other patient who developed IDH-DS (fever, pleural effusion, pulmonary infiltrates on imaging) on D31 of treatment and was managed with steroids. No patient died due to IDH-DS.

\section{DISCUSSION}

Treatment options remain limited in patients with $A M L$, particularly those patients not eligible for intensive chemotherapy or in patients with relapsed/refractory disease. The presence of an IDH2 mutation identifies important treatment options including targeted mutant IDH2i therapy. In ND IDH2 ${ }^{\text {mut }} \mathrm{AML}$, we report a $\mathrm{CRC}$ of $100 \%$ with $85 \%$ CR with ENA + AZA and at a median follow-up of 13.1 months, the median OS was not reached. Notably four patients received ENA + AZA + VEN triplet combination. The outcomes of ENA + AZA are in line with azacitidine and venetoclax (84\% CRc, median OS - not reached) combination [24], as well as the outcomes of ENA + AZA in a randomized phase 2 trial with $C R$ c rate $63 \%$ and median OS 22 months in patients with ND IDH2 ${ }^{\text {mut }}$ AML [25].

$\mathrm{R} / \mathrm{R}$ AML is notoriously treatment-resistant as shown by an ORR of $14-28 \%$ in a study evaluating three different treatment regimens in patients with primary refractory disease or in first relapse [26]. In our $\mathrm{R} / \mathrm{R} I \mathrm{IDH} 2^{\text {mut }} \mathrm{AML}$ cohort that mostly included heavily pretreated patients, we report a CRC of $58 \%$, with $28 \%$ CR

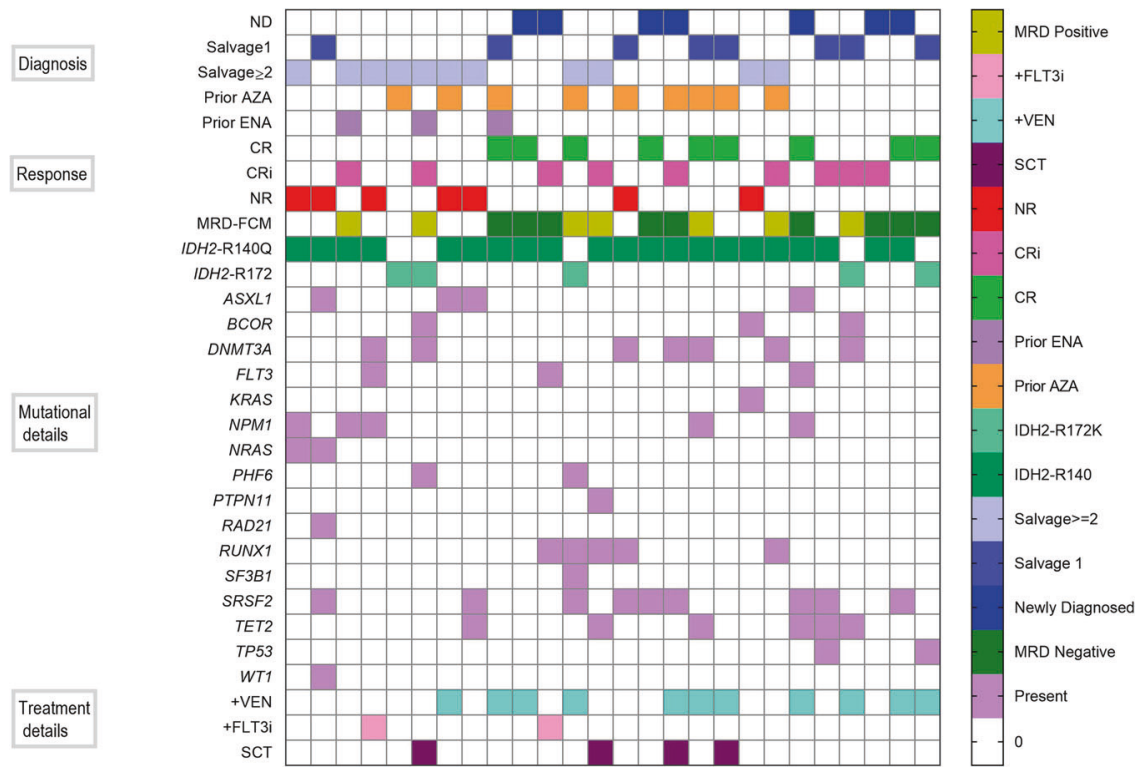

Fig. 4 Landscape of entire cohort with genomics and outcomes. Mutational landscape of the whole cohort. 
Table 3. Treatment emergent adverse events in newly diagnosed and relapsed/refractory patients with $I D H 2$ mutant acute myeloid leukemia.

\begin{tabular}{|lll|}
\hline TEAE & $\begin{array}{l}\text { Newly diagnosed } \\
\boldsymbol{n}=\mathbf{7}\end{array}$ & $\begin{array}{l}\text { Relapsed/refractory } \\
\boldsymbol{n}=\mathbf{1 9}\end{array}$ \\
\hline IDH-DS & $1(14)$ & $1(5)$ \\
\hline Febrile neutropenia & $1(14)$ & $5(26)$ \\
$\geq$ Gr 3 & & $1(5)$ \\
\hline ALT $\geq$ Gr 3 & 0 & $1(5)$ \\
\hline AST $\geq$ Gr 3 & 0 & $7(37)$ \\
\hline Total bilirubin $\geq G r 3$ & $2(29)$ & \\
\hline GI (any grade) & & $1(5)$ \\
\hline Nausea & 0 & $2(11)$ \\
\hline Vomiting & 0 & $4(21)$ \\
\hline Diarrhea & 0 &
\end{tabular}

TEAE- Treatment emergent adverse event, IDH-DS- IDH inhibitor induced differentiation syndrome, AST- Aspartate aminotransferase, ALT- Alanine aminotransferase, Gl- Gastrointestinal

All results expressed as No. (\%) or median [Minimum - maximum], unless specified.

with ENA + AZA combination. Patients in first relapse had numerically higher CRc and a significantly better OS than those in second relapse or later. This significant survival benefit seen among patients in first relapse suggest an earlier therapeutic role for ENA + AZA combination in patients with R/R IDH2 ${ }^{\text {mut }} A M L$. This is consistent with the data from ENA monotherapy, where both response rates and OS decreased in more heavily pretreated patients [22].

The ENA + AZA + VEN "triplet" combination in R/R IDH2 ${ }^{\text {mut }} A M L$ was well tolerated and patients with prior HMA or ENA continued either of these agents with VEN. ENA + AZA + VEN triplet combination demonstrated a CRc of $86 \%$ even in those with prior exposure to AZA or ENA. The median OS was not reached, and the 1 -year OS was $67 \%$. Encouraging outcomes with ENA + AZA + VEN compare favorably to decitabine-10 days and venetoclax combination with $\mathrm{CR} / \mathrm{CRi}$ rate of $54 \%$ and median OS of 14.7 months in $\mathrm{R} / \mathrm{R} I D H 2^{\text {mut }} \mathrm{AML}$ [27].

While responding patients did demonstrate modest reductions in the IDH2 VAF compared to non-responding patients, "clearance" of the $I D H 2$ mut to $<1 \%$ was not identified in any responding patient at the time of this analysis. This is consistent with prior reports stating response is not dependent on molecular clearance, and in line with data from the ENA + AZA vs AZA alone trial for newly diagnosed older $A M L$, where the median maximum $I D H 2$ VAF reduction was $81.5 \%$ and occurred in responding patients with a median of 6 cycles of the combination [25]. Additionally, given the enrichment of MDS-type mutations in this cohort, it is likely the IDH2 remains as persistent of a "CHIP" clone in many of these patients, despite clearance of the AML. Whether deeper responses are associated with more durable and prolonged remissions is an important question for future follow up. Additionally, concomitant receptor tyrosine kinase mutations and TP53 mutations were associated with primary and secondary resistance in the relapsed $I D H 2^{\text {mut }}$ population as has been previously described $[22,23]$. While TET2 mutations are uncommon in the setting of $I D H 2$ mutations due to biologically overlapping pathogenic mechanisms [28], in our cohort, five (20\%) patients harbored TET2 co-mutations, and all of them were responders. Whether these mutations were found in the same or different leukemic clones in these patients is not known.

ENA + AZA combination was generally well tolerated. The most common non-hematologic toxicity was transient indirect hyperbilirubinemia (35\%) that did not warrant treatment interruption, which is a known side effect of enasidenib due to UGT1A1 metabolism. Early mortality rate in ND patients was $0 \%$ and was $5 \%$ for R/R group, which was reassuring $[9,10]$. ENA is known to cause differentiation syndrome (IDH-DS) by virtue of its activity as a differentiating agent. IDH-DS occurred with anticipated frequencies, and was lower with ENA + AZA (8\%) than ENA monotherapy (11.7\%) [29]. Among the two patients who developed IDH-DS, one patient received concurrent gilteritinib which has also been known to cause differentiation syndrome [30]. Prompt initiation of steroids and supportive measures rapidly mitigated the signs and symptoms of IDH-DS. The safety profile of ENA + AZA combination was comparable to ENA monotherapy [29] and no new TEAEs were observed.

The limitations of our study include a lack of comparator arm and the inherent risk of selection bias. To minimize selection bias, we enrolled consecutive $I D H 2^{\text {mut }} \mathrm{AML}$ patients who met the study eligibility criteria, and the eligibility criteria allowed prior therapies (including ENA or HMA or VEN) and/or antecedent hematologic neoplasms and ECOG PS up to 3, to try to replicate a more "real world" patient population.

In summary, ENA + AZA is a safe and effective treatment combination, both for ND elderly pts, as well as in R/R AML, and even those patients having previously received prior HMA or ENA. This pilot evaluation of ENA + AZA in R/R IDH2 ${ }^{\text {mut }} A M L$ suggests an earlier therapeutic role of ENA, with improved responses seen in patients treated during their first relapse. Furthermore, the "triplet" of ENA + AZA + VEN appears to be improve outcomes in high risk, heavily pretreated R/R IDH2 ${ }^{\text {mut }} A M L$. Ongoing systematic evaluation of an all oral regimen incorporating decitabine/ cedazuridine, enasidenib and venetoclax may confirm the activity of this combination in R/R IDH2 ${ }^{\text {mut }}$ AML. (NCT04774393)

\section{REFERENCES}

1. Al-Ali HK, Jaekel N, Junghanss C, Maschmeyer G, Krahl R, Cross M, et al. Azacitidine in patients with acute myeloid leukemia medically unfit for or resistant to chemotherapy: a multicenter phase I/II study. Leuk Lymphoma. 2012;53:110-7.

2. Maurillo L, Venditti A, Spagnoli A, Gaidano G, Ferrero D, Oliva E, et al. Azacitidine for the treatment of patients with acute myeloid leukemia: report of 82 patients enrolled in an Italian Compassionate Program. Cancer 2012;118:1014-22.

3. Thépot S, Itzykson R, Seegers V, Recher C, Raffoux E, Quesnel B, et al. Azacitidine in untreated acute myeloid leukemia: a report on 149 patients. Am J Hematol. 2014;89:410-6.

4. Itzykson R, Thépot S, Berthon C, Delaunay J, Bouscary D, Cluzeau T, et al. Azacitidine for the treatment of relapsed and refractory $A M L$ in older patients. Leuk Res. 2015;39:124-30.

5. DiNardo CD, Jonas BA, Pullarkat V, Thirman MJ, Garcia JS, Wei AH, et al. Azacitidine and Venetoclax in Previously Untreated Acute Myeloid Leukemia. N. Engl J Med. 2020;383:617-29.

6. Short NJ, Rytting ME, Cortes JE. Acute myeloid leukaemia. Lancet 2018;392:593-606.

7. DiNardo CD, Ravandi F, Agresta S, Konopleva M, Takahashi K, Kadia T, et al. Characteristics, clinical outcome, and prognostic significance of IDH mutations in AML. Am J Hematol. 2015;90:732-6.

8. Ward PS, Patel J, Wise DR, Abdel-Wahab O, Bennett BD, Coller HA, et al. The common feature of leukemia-associated IDH1 and IDH2 mutations is a neomorphic enzyme activity converting alpha-ketoglutarate to 2-hydroxyglutarate. Cancer Cell. 2010;17:225-34.

9. Stein EM, DiNardo CD, Pollyea DA, Fathi AT, Roboz GJ, Altman JK, et al. Enasidenib in mutant IDH2 relapsed or refractory acute myeloid leukemia. Blood 2017;130:722-31.

10. Pollyea DA, Tallman MS, de Botton S, Kantarjian HM, Collins R, Stein AS, et al. Enasidenib, an inhibitor of mutant IDH2 proteins, induces durable remissions in older patients with newly diagnosed acute myeloid leukemia. Leukemia 2019;33:2575-84.

11. Chopra VS, Avanzino B, Mavrommatis K, Olshen A, DiMartino J, MacBeth KJ. Functional characterization of combining epigenetic modifiers azacitidine and AG-221 in the TF-1: IDH2R140Q AML model. AACR; 2016.

12. Courtney DiNardo AS, Eytan Stein, Pau Montesinos, Andrew Wei, Stéphane de Botton, Amer Zeidan, Amir Fathi, Lynn Quek, Hagop Kantarjian, Mark Frattini, Frederik Lersch, Jing Gong, Aleksandra Franovic, Paresh Vyas, Hartmut Döhner. 
ENASIDENIB PLUS AZACITIDINE SIGNIFICANTLY IMPROVES COMPLETE REMISSION AND OVERALL RESPONSE RATES VERSUS AZACITIDINE MONOTHERAPY IN MUTANT-IDH2 NEWLY DIAGNOSED ACUTE MYELOID LEUKEMIA (ND-AML). EHA2020.

13. Cathelin S, Sharon D, Subedi A, Cojocari D, Phillips DC, Leverson JD, et al. Combination of Enasidenib and Venetoclax Shows Superior Anti-Leukemic Activity Against IDH2 Mutated AML in Patient-Derived Xenograft Models. Blood 2018;132:562

14. Cathelin S, Sharon D, Subedi A, Cojocari D, Phillips DC, Leverson JD, et al. Enasidenib-induced differentiation promotes sensitivity to venetoclax in IDH2mutated acute myeloid leukemia. Leukemia. 2021. 10.1038/s41375-021-01468-y.

15. Boddu P, Kantarjian HM, Garcia-Manero G, Ravandi F, Verstovsek S, Jabbour E, et al. Treated secondary acute myeloid leukemia: a distinct high-risk subset of AML with adverse prognosis. Blood Adv. 2017;1:1312-23.

16. Daver N, Schlenk RF, Russell NH, Levis MJ. Targeting FLT3 mutations in AML: review of current knowledge and evidence. Leukemia 2019;33:299-312.

17. DiNardo CD, Maiti A, Rausch CR, Pemmaraju N, Naqvi K, Daver NG, et al. 10-day decitabine with venetoclax for newly diagnosed intensive chemotherapy ineligible, and relapsed or refractory acute myeloid leukaemia: a single-centre, phase 2 trial. The Lancet Haematology.

18. Xu J, Jorgensen JL, Wang SA. How Do We Use Multicolor Flow Cytometry to Detect Minimal Residual Disease in Acute Myeloid Leukemia? Clin Lab Med. 2017;37:787-802.

19. Ok CY, Loghavi $S$, Sui $D$, Wei $P$, Kanagal-Shamanna $R$, Yin $C C$, et al. Persistent IDH1/2 mutations in remission can predict relapse in patients with acute myeloid leukemia. Haematologica 2019;104:305-11.

20. Thall PF, Simon RM, Estey EH. Bayesian sequential monitoring designs for singlearm clinical trials with multiple outcomes. Stat Med. 1995;14:357-79.

21. Döhner H, Estey E, Grimwade D, Amadori S, Appelbaum FR, Büchner T, et al. Diagnosis and management of AML in adults: 2017 ELN recommendations from an international expert panel. Blood 2017;129:424-47.

22. Stein EM, DiNardo CD, Fathi AT, Pollyea DA, Stone RM, Altman JK, et al. Molecular remission and response patterns in patients with mutant-IDH2 acute myeloid leukemia treated with enasidenib. Blood 2019;133:676-87.

23. Wang F, Morita K, DiNardo CD, Furudate K, Tanaka T, Yan Y, et al. Leukemia stemness and co-occurring mutations drive resistance to IDH inhibitors in acute myeloid leukemia. Nat Commun. 2021;12:2607.

24. Pollyea DA, Dinardo CD, Arellano ML, Pigneux A, Fiedler W, Konopleva M, et al. Results of Venetoclax and Azacitidine Combination in Chemotherapy Ineligible Untreated Patients with Acute Myeloid Leukemia with IDH 1/2 Mutations. Blood 2020;136:5-7.

25. DiNardo $C D$, Schuh $A C$, Stein EM, Montesinos $P$, Wei $A H$, de Botton $S$, et al. Enasidenib plus azacitidine versus azacitidine alone in patients with newly diagnosed, mutant-IDH2 acute myeloid leukaemia (AG221-AML-005): a singlearm, phase $1 \mathrm{~b}$ and randomised, phase 2 trial. Lancet Oncol. 2021;22:1597-608.

26. Litzow MR, Wang XV, Carroll MP, Karp JE, Ketterling RP, Zhang Y, et al. A randomized trial of three novel regimens for recurrent acute myeloid leukemia demonstrates the continuing challenge of treating this difficult disease. Am J Hematol. 2019;94:111-7.

27. Venugopal S, Maiti A, DiNardo CD, Loghavi S, Daver NG, Kadia TM, et al. Decitabine and venetoclax for IDH1/2-mutated acute myeloid leukemia. Am J Hematol. 2021;96:E154-E7.

28. Figueroa ME, Abdel-Wahab O, Lu C, Ward PS, Patel J, Shih A, et al. Leukemic IDH1 and IDH2 mutations result in a hypermethylation phenotype, disrupt TET2 function, and impair hematopoietic differentiation. Cancer Cell. 2010;18:553-67.

29. Fathi AT, DiNardo CD, Kline I, Kenvin L, Gupta I, Attar EC, et al. Differentiation Syndrome Associated With Enasidenib, a Selective Inhibitor of Mutant Isocitrate Dehydrogenase 2: Analysis of a Phase 1/2 Study. JAMA Oncol. 2018:4:1106-10.

30. McMahon CM, Canaani J, Rea B, Sargent RL, Qualtieri JN, Watt CD, et al. Gilteritinib induces differentiation in relapsed and refractory FLT3-mutated acute myeloid leukemia. Blood Adv. 2019;3:1581-5.

\section{ACKNOWLEDGEMENTS}

This study was supported in part by the Brystol Meyer Squibb. We thank the patients, their caregivers, and members of the study team involved in this trial. SV: None. KT: has received personal fees for service on advisory boards of Symbio Pharmaceuticals, GSK Celgene. NGD: reports grant from Abbvie, Genentech, Astellas, Daiichi-Sankyo, Pfizer, BMS, Immunogen, Novimmune, Forty-seven; personal fees from Abbvie, Genentech Astellas, Daiichi-Sankyo, Pfizer, BMS, Immunogen, Jazz pharmaceuticals, Trillium, Fortyseven, Gilead, Kite, Novartis. GB: SL: None. NJS: reports grant from Takeda Oncology, Astellas; Personal fees from Takeda Oncology, AstraZeneca, Amgen. MO: None. LM: Gl: CBR: MY: None. TMK: None. MA: FR: None. MYK: has received grants from $\mathrm{NIH}, \mathrm{NCl}$ Abbvie, Genentech, Stemline Therapeutics, Forty-Seven, Eli Lilly, Cellectis, Calithera Ablynx, Astra Zeneca; Consulting/honorarium from AbbVie, Genentech, F. Hoffman La-Roche, Stemline Therapeutics, Amgen, Forty-Seven, Kisoji; clinical trial support from Ascentage; stocks/royalties in Reata Pharmaceutical. HMK: grants and other from AbbVie, grants and other from Agios, grants and other from Amgen, grants from Ariad grants from Astex, grants from BMS, from Cyclacel, grants from Daiichi-Sankyo, grants and other from Immunogen, grants from Jazz Pharma, grants from Novartis, grants and other from Pfizer, other from Actinium, other from Takeda, outside the submitted work. CDD: Personal fees from Abbvie, personal fees from Agios, personal fees from Novartis, personal fees from ImmuneOnc, personal fees from Daiichi Sankyo, personal fees from Celgene, personal fees from Jazz, personal fees from Notable Labs, outside the submitted work. This paper was presented as a poster in the European Hematology Association Annual Meeting, virtual June 9-17, 2021.

\section{AUTHOR CONTRIBUTIONS}

Conception and design: CDD, KT, FR, MK. Provision of study materials or patients: KT, ND, AM, GB, SL, NJS, MO, LM, GCl, BSR, MY, TK, MA, FR, MK, HMK, CDD. Collection and assembly of data: SV, KT, ND, AM, GB, SL, NJS, MO, LM, GCI, BSR, MY, TK, MA, FR, MK, HMK, CDD.

\section{COMPETING INTERESTS}

The authors declare no competing interests.

\section{ADDITIONAL INFORMATION}

Correspondence and requests for materials should be addressed to Courtney D. DiNardo.

Reprints and permission information is available at http://www.nature.com/ reprints

Publisher's note Springer Nature remains neutral with regard to jurisdictional claims in published maps and institutional affiliations.

Open Access This article is licensed under a Creative Commons Attribution 4.0 International License, which permits use, sharing, adaptation, distribution and reproduction in any medium or format, as long as you give appropriate credit to the original author(s) and the source, provide a link to the Creative Commons license, and indicate if changes were made. The images or other third party material in this article are included in the article's Creative Commons license, unless indicated otherwise in a credit line to the material. If material is not included in the article's Creative Commons license and your intended use is not permitted by statutory regulation or exceeds the permitted use, you will need to obtain permission directly from the copyright holder. To view a copy of this license, visit http://creativecommons. org/licenses/by/4.0/.

(c) The Author(s) 2022 